Results The PAHO website includes all age group fatal drowning data for $45 / 53$ countries and territories. Drowning is a top 5 cause of all deaths in $42 / 45$ countries and territories, in at least one age group. Drowning is the lead cause of death in 15/45 nations, in at least one age group. The greatest burden of fatal drowning is children and youth. Fatal drowning rates are greatest in LMICs.

Conclusions Fatal Drowning is a significant public health issue in the WHO Region of the Americas/Pan American Health Organization. Drowning Prevention strategies should become priority public health interventions in all countries and territories, with a focus on children and youth.

Learning The WHO Region of the Americas/PAHO should develop a program for drowning prevention focused on proven interventions for high burden countries.

\section{B.002 SERIOUSLY GUYS? YOUNG MALES AND COASTAL DROWNING DEATHS IN AUSTRALIA}

Jaz Lawes*, Luke Strasiotto, Shane Daw. Surf Life Saving Australia, Sydney, Australia

\subsection{6/injuryprev-2021-safety.38}

Males are consistently overrepresented in fatal coastal drowning incidents, with young males representing a significant proportion of these. This is often attributed to their greater participation and increased confidence levels which do not necessarily reflect abilities.

Using annual coastal safety surveys and the national coastal fatality database, this study examined characteristics of drowning deaths and current perceptions of coastal safety within this high-risk demographic. Inflated confidence levels of young males to engage in risk-taking behaviour was confirmed with survey respondents consistently considering themselves to be experienced enough to take risks during activities in comparison to the total adult population.

Since 2004, males aged between 16-39 account for $36 \%$ of all drowning deaths. In 2018-19, 44 drowning deaths were young males. Average fatality rate ratios revealed young males are twice as likely $(2.05: 1)$ to drown on our coast, particularly at rock/cliff and beach locations. Young males were more likely to drown while jumping, snorkelling, swimming and wading, with $58 \%$ of young male drowning victims reported to have little to no experience in the activity being undertaken at the time of death. Analyses revealed young males were twice as likely to drown on a public holiday and significantly more likely to have consumed alcohol or drugs $(\mathrm{RR}=1.3$; CI:1.1-1.5) than other demographics.

These results highlight that the adventurous nature and overconfidence exhibited by young males puts them at significant risk of drowning and should be a target for future drowning prevention strategies on the Australian coast.

\section{B.003 WHAT MAKES A POPULATION 'HIGH-RISK' FOR DROWNING IN AUSTRALIA?}

\footnotetext{
1,2 Stacey Willcox-Pidgeon* ${ }^{*}{ }^{1,2}$ Richard Franklin, ${ }^{2}$ Peter Leggat, ${ }^{2}$ Sue Devine, ${ }^{1}$ Justin Scarr. ${ }^{1}$ Royal Life Saving Society - Australia, Sydney, Australia; ${ }^{2}$ James Cook University, Townsville, Australia
}

10.1136/injuryprev-2021-safety.39
Background In Australia, people born overseas are considered at higher-risk of drowning. Common perceptions is that being from overseas (including tourists), with English as another language makes someone more vulnerable to drowning. The aims were to analyse drowning deaths of people born overseas, and to identify specific risk factors to better inform prevention strategies targeted to migrant populations.

Methods A total population analysis of all unintentional, drowning deaths over 10 years (2008/09-2017/18) of people born outside of Australia were included. Cases were extracted from the Royal Life Saving National Fatal Drowning Database, collated from the National Coronial Information System. Descriptive statistics, relative risk and crude drowning rates were calculated.

Results 794 people drowned, who were born overseas, accounting for $27 \%$ of total drowning deaths. $77 \%$ were men, 24\% were aged 25-34 years. Three groups were identified: Australian residents (83\%), overseas visitors (14\%) and international students (4\%). Differences in drowning rates were found when analysed by country of birth, and time in Australia e.g. new arrivals $0-5$ years versus resident for 5-10 years, 10-20 years and 20 years + .

Conclusion These results identified that distinct sub-groups exist within the 'high-risk' population category, each with differing risk factors for drowning. These findings offer practical insights when devising effective drowning prevention policy and programs for specific groups. This study informed the Australian Water Safety Strategy 2030 area pertaining to 'highrisk' populations.

Learning Outcomes 'High-risk' populations for drowning are not homogenous and this should be taken into account when developing drowning prevention policies and strategies

\section{B.004 ANOTHER GENDER DATA GAP: WOMENS DROWNING IN NEW ZEALAND}

Karen Wells*. Water Safety New Zealand, Wellington, New Zealand

\subsection{6/injuryprev-2021-safety.40}

Background There is currently a paucity of literature regarding female drowning worldwide. The majority of research on drowning discusses female drowning as a secondary topic to male drowning. Drowning fatalities are predominantly males in New Zealand which has potentially led to a lack of focus on female drowning. This research is the first of its kind in New Zealand.

Method This research first outlines the breadth and consequences of the gender data gap. Analysis of DrownBase (New Zealand's drowning data base) data from the last 40 years has been undertaken. This data analysis focused on trends in female drowning data over the last 40 years to identify the risk factors relating to female drowning and how they have changed.

Results Results show that the issue of female fatal and nonfatal drowning is a growing problem. Female drownings are rising in New Zealand, with 20\% more fatalities and a $30 \%$ increase in non-fatal drownings in 2018 compared to the 2013-2017 five-year average. Female drowning differs to male drowning, in $201861 \%$ of female fatalities were a result of accidental immersions (in contrast to $35 \%$ of male fatalities). 